\title{
Assessment of Myocardial Viability Using Nuclear Medicine Imaging in Dextrocardia
}

\author{
Shwetal U. Pawar, Suruchi S. Shetye, Mangala K. Ghorpade, and Rahul Azeez Seena \\ Department of Nuclear Medicine, King Edward Memorial Hospital, Seth G.S. Medical College, Mumbai, India
}

Imaging of dextrocardia in humans requires an understanding of the orientation of the heart chambers and walls. There are many types of cardiac malpositioning, such as dextrocardia (with or without situs inversus), mesocardia, and levocardia. Myocardial perfusion scintigraphy of dextrocardia has been explained in case reports and imaging atlases; however, myocardial viability assessment using nuclear medicine imaging techniques is less documented in the literature. Methods: In 2 cases of dextrocardia with situs inversus and 1 case of mesocardia, myocardial viability was assessed using ${ }^{99 m}$ Tc-sestamibi rest perfusion scintigraphy and ${ }^{18} \mathrm{~F}-\mathrm{FDG}$ PET. Cardiac SPECT images of dextrocardia with situs inversus were acquired using the feet-first supine position with a $180^{\circ}$ arc from left anterior oblique to right posterior oblique, whereas a rightlateral-to-left-lateral arc was used for mesocardia. The processing and reconstruction were done by entering the dataset for the feetfirst supine position and repeating after entering the dataset for the feet-first prone position. The 2 sets of reconstructed images were compared for orientation of walls and cardiac chambers. Results: The first processing, using the feet-first supine position, revealed an interchanged septum and lateral wall in reconstructed images of dextrocardia with situs inversus. This interchange was corrected by changing the position to prone during processing of the rest perfusion and PET raw data. The display of cardiac slices in various axes matched the conventional nomenclature for the septum and lateral wall, leading to easy interpretation. However, this change was not required in the mesocardia, for which the location of the heart chambers was not interchanged. Conclusion: Because the acquisition protocol for SPECT is a semicircular orbit, the various types of dextrocardia require careful selection of the arc, with the patient positioning kept feet-first supine. Processing and reconstruction of data by changing the patient position to prone was found to be most useful method of matching the septum and lateral wall orientation for interpretation of images.

Key Words: human dextrocardia; myocardial viability; rest perfusion

J Nucl Med Technol 2020; 48:372-377

DOI: 10.2967/jnmt.120.248435

\footnotetext{
Received Apr. 30, 2020; revision accepted Jun. 26, 2020.

For correspondence or reprints contact: Shwetal U. Pawar, Department of Nuclear Medicine, King Edward Memorial Hospital, Seth G.S. Medical College, Parel, Mumbai, Maharashtra, India 400012.

E-mail: shwetal13@yahoo.com

Published online Sep. 4, 2020.

COPYRIGHT (c) 2020 by the Society of Nuclear Medicine and Molecular Imaging.
}

D extrocardia, a rare cardiac malposition in which the heart is positioned in the right hemithorax, occurs in approximately 1 in 12,000 people. The term cardiac malposition implies that the heart is located anywhere other than its usual position in the left hemithorax, or it may describe a situation in which the heart is located in the left hemithorax but other organs are in abnormal positions, as in situs inversus viscerum. Dextrocardia, levocardia, and mesocardia are general terms that indicate cardiac position only and not intracardiac anatomy. Dextrocardia denotes a right-sided heart, levocardia a left-sided heart, and mesocardia a midline heart (1).

Arcilla et al. (2) classified dextrocardia into 5 types. In type I (mirror-image dextrocardia), the anatomic right atrium and right ventricle are situated to the left of and anterior to the corresponding systemic chambers. A mirror-image arrangement of the cardiac chambers is present since the frontal relationship of the chambers is reversed, yet the anteroposterior arrangement is normal. In type II (dextroversion complex), the relations of the cardiac chambers are normal; the right atrium and right ventricle are situated to the right of and posterior to the corresponding systemic chambers. In complete dextroversion, the cardiac apex is situated anteriorly and to the right; in incomplete dextroversion or mesoversion, it is in the substernal region and the longitudinal axis of the heart is parallel to the midsagittal axis of the chest. Type III dextrocardia (mixed dextrocardia) is characterized by inversion of the atria alone or of the ventricles alone. The arrangement of the cardiac chambers is therefore somewhat similar to that of types I and II. In type IV (congenital dextroposition), the heart is in the mid chest, but the arrangement of the chambers is normal and the cardiac apex is still directed to the left and anteriorly. Types I-IV represent the intrinsic group of dextrocardia, since the cardiac heterotaxy is caused by a developmental anomaly of the primitive heart tube. In type $\mathrm{V}$ (congenital extrinsic), the abnormal position of the heart is due to its displacement by congenital anomalies of the lungs, diaphragm, or chest cage. The rightward displacement may be in the frontal plane only (simple dextroposition) or may occur in both the frontal and the horizontal planes (dextroposition with pivotal rotation). 
Patients with dextrocardia may also have coronary artery disease similar to that in the general population and would need similar investigations. Myocardial perfusion scintigraphy (MPS) and myocardial viability assessment using ${ }^{18} \mathrm{~F}-\mathrm{FDG}$ PET are 2 noninvasive procedures to diagnose or evaluate coronary artery disease and assess myocardial viability, respectively.

Since dextrocardia is a rare clinical condition, general experience regarding acquisition, processing, and interpretation of cardiac SPECT is limited, with only a few reports existent in the literature on MPS and even fewer reports on myocardial viability assessment. Because of the unusual orientation of the heart in the hemithorax in patients with dextrocardia, the usual acquisition protocols would require modification. However, even if acquisitions were to be based on the anatomic differences in dextrocardia, difficulties might arise during data processing and analysis with the software currently available for $\gamma$-cameras. These difficulties make cardiac SPECT studies in patients with dextrocardia even more challenging.

Many case reports have provided guidance about positioning and image processing using MPS in dextrocardia patients (3-9); however, there are fewer reports on assessing myocardial viability using ${ }^{18} \mathrm{~F}-\mathrm{FDG}$ PET.

${ }^{18}$ F-FDG PET myocardial viability studies include rest MPS and ${ }^{18} \mathrm{~F}-\mathrm{FDG}$ PET imaging, each requiring different acquisition methods. The purpose of this case series study was to assess different methods of reconstruction and processing in order to understand how best to orient the heart chambers and walls for interpretation of the various types of dextrocardia.

\section{CASE REPORTS}

This was a retrospective study of dextrocardia patients referred to the nuclear medicine department of Seth G.S. Medical College and King Edward Memorial Hospital, Mumbai, India, from January 2015 to December 2018. The study included patients with various types of dextrocardia who were referred to the department for assessment of myocardial viability. Patients who had undergone surgery or coronary-intervention treatment were excluded from the data. This retrospective study was approved by the institutional ethics committee, and the need for patient consent was waived. Three patients were included (Table 1).

\section{Patient 1}

A 66-y-old hypertensive man presented with retrosternal discomfort on exertion and excessive perspiration. He was diagnosed with non-ST-segment elevation myocardial infarction (Fig. 1A) and had an elevated level of creatinine kinase. Chest radiography and 2-dimensional echocardiography revealed dextrocardia, concentric left ventricular hypertrophy, and a left ventricular ejection fraction of $60 \%$.

Coronary angiography demonstrated a $90 \%$ lesion in the mid section of the left anterior descending (LAD) coronary artery and an ostial total occlusion in the obtuse marginal territory. The left main, the rest of the left circumflex, and

TABLE 1

Patient Details Regarding Dextrocardia, Acquisition, and Processing

\begin{tabular}{|c|c|c|c|}
\hline Variable & Patient 1 & Patient 2 & Patient 3 \\
\hline Age $(y)$ & 66 & 61 & 42 \\
\hline Sex & M & $\mathrm{F}$ & M \\
\hline Presentation & NSTEMI & Breathlessness & Chest pain \\
\hline Type of dextrocardia & Complete & Complete & Incomplete (mesocardia) \\
\hline Situs inversus? & Yes & Yes & No \\
\hline \multicolumn{4}{|l|}{ Patient positioning } \\
\hline SPECT & Feet-first supine & Feet-first supine & Feet-first supine \\
\hline 18F-FDG PET & Head-first supine & Head-first supine & Head-first supine \\
\hline \multicolumn{4}{|c|}{ Imaging arc during acquisition } \\
\hline SPECT & $\begin{array}{l}45^{\circ} \text { LAO to } 135^{\circ} \\
\text { RPO clockwise }\end{array}$ & $\begin{array}{l}45^{\circ} \text { LAO to } 135^{\circ} \\
\text { RPO clockwise }\end{array}$ & $\begin{array}{l}180^{\circ} \text { right lateral to } \\
\text { left lateral }\end{array}$ \\
\hline${ }^{18} \mathrm{~F}-\mathrm{FDG}$ PET & $360^{\circ}$ & $360^{\circ}$ & $360^{\circ}$ \\
\hline \multicolumn{4}{|l|}{ Routine processing* } \\
\hline SPECT & Feet-first supine & Feet-first supine & Feet-first supine \\
\hline${ }^{18} \mathrm{~F}-\mathrm{FDG}$ PET & Head-first supine & Head-first supine & Head-first supine \\
\hline Cardiac wall orientation & $\begin{array}{l}\text { Interchanged septum } \\
\text { and lateral wall }\end{array}$ & $\begin{array}{l}\text { Interchanged septum } \\
\text { and lateral wall }\end{array}$ & Not affected \\
\hline Change in processing & Required & Required & Not required \\
\hline SPECT & Feet-first prone & Feet-first prone & - \\
\hline${ }^{18}$ F-FDG PET & Head-first prone & Head-first prone & - \\
\hline Cardiac wall orientation & Corrected & Corrected & - \\
\hline
\end{tabular}




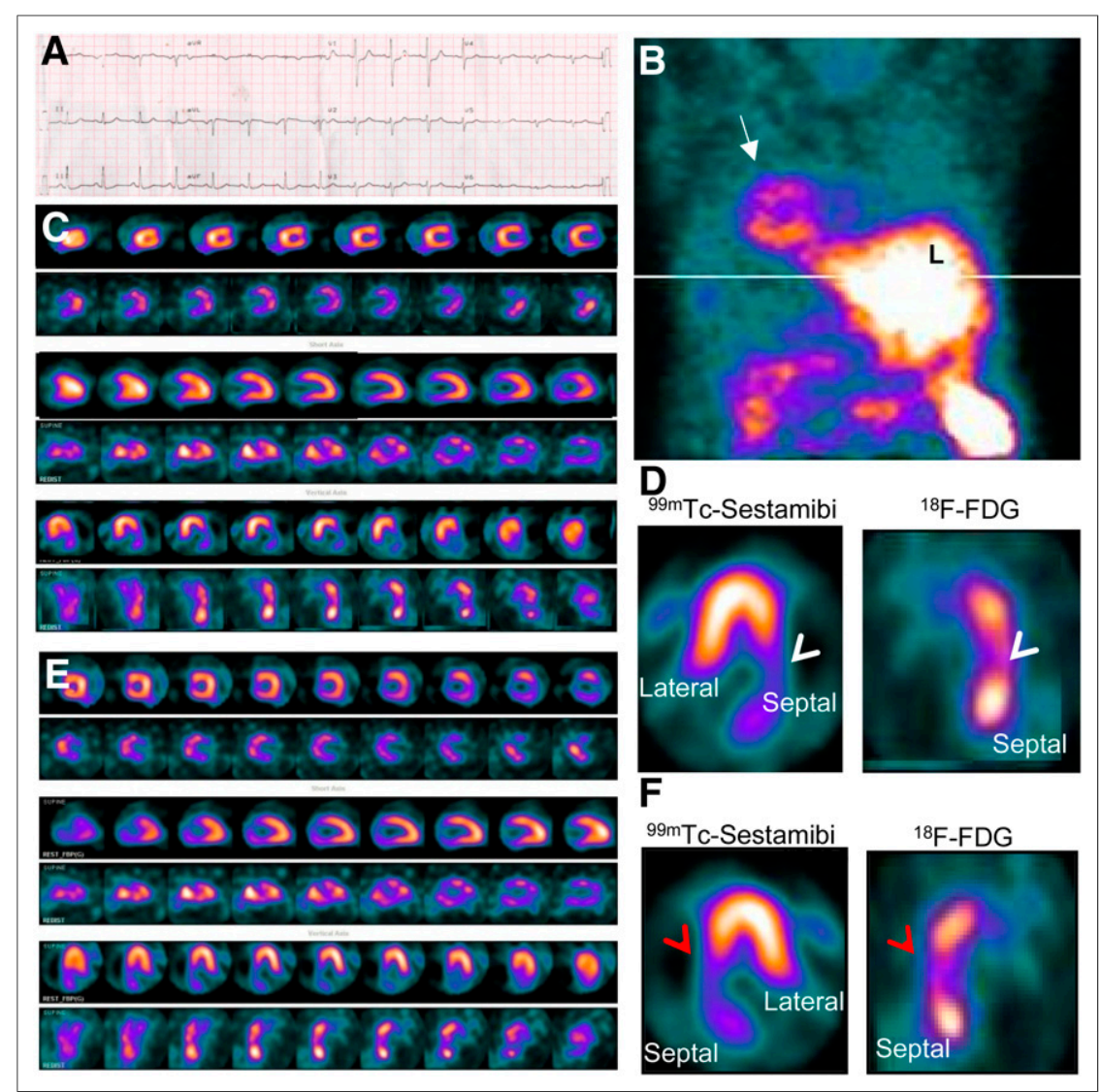

FIGURE 1. A 66-y-old man who had dextrocardia with situs inversus presented with non-ST-segment elevation myocardial infarction. (A) Poor R-wave progression was seen on V3-V6 chest leads on electrocardiography, which was obtained with chest leads arranged on right chest wall. (B) Maximum-intensity-projection rest perfusion SPECT image showed heart in right chest (arrow) and liver on left side. (C and D) Processing of raw data in feet-first supine position showed interchanged lateral wall and septum in reconstructed horizontal long-axis image (arrowheads). ( $E$ and $F$ ) Orientation of septum and lateral wall was corrected in repeat processing, which was done after entering dataset for feet-first prone position instead of feet-first supine to match conventional nomenclature of display image (arrowheads) Perfusion was absent from mid-anteroseptal, basal anteroseptal, mid-inferoseptal, and basal inferoseptal segments whereas ${ }^{18} \mathrm{~F}-\mathrm{FDG}$ uptake was present in same segments, thus suggesting viable myocardium (matched perfusion and metabolism).

the right coronary arteries were normal. The patient was referred to the nuclear medicine department for evaluation of myocardial viability in the LAD and obtuse marginal territories.

The myocardial viability study consisted of 2 parts: resting MPS followed by ${ }^{18} \mathrm{~F}-\mathrm{FDG}$ cardiac PET. Resting MPS was performed 45 min after intravenous injection of $740 \mathrm{MBq}(20 \mathrm{mCi})$ of ${ }^{99 \mathrm{~m}} \mathrm{Tc}$-sestamibi at rest. Images were acquired on dual-head $\gamma$-camera (Infinia Hawkeye; Wipro GE Healthcare) with a low-energy high-resolution collimator in L-mode, a feet-first supine position, and a clockwise direction from $45^{\circ}$ left anterior oblique (LAO) to $135^{\circ}$ right posterior oblique (RPO) (10). On the same day, cardiac PET was performed after intravenous injection of $185 \mathrm{MBq}(5$ $\mathrm{mCi}$ ) of ${ }^{18} \mathrm{~F}-\mathrm{FDG}$ at rest after ensuring a fasting blood sugar level of less than $140 \mathrm{mg} / \mathrm{dl}$. Images were acquired $60 \mathrm{~min}$ after injection with the patient supine on a PET/CT machine (Discovery 710; Wipro GE Healthcare). The scan was acquired in 3-dimensional static mode at 1 bed position for $10 \mathrm{~min}$. The images were reconstructed using an iterative reconstruction algorithm (11). The rest perfusion and ${ }^{18} \mathrm{~F}$-FDG PET images were processed and reconstructed using Emory Cardiac Toolbox, version 3.0.

The maximum-intensity-projection image demonstrated dextrocardia with situs inversus (Fig. 1B). Since this patient had mirror-image dextrocardia, the positions of the septum and lateral walls were interchanged. Also, the right ventricle was to the left of the left ventricle. Thus, the SPECT and PET reconstructed display images with the heart positioned in the given acquisition parameters showed the interchanged septum and lateral walls more clearly in the horizontal long-axis and short-axis slices (Figs. $1 \mathrm{C}$ and 1D). A second reconstruction was done by selecting the prone position for the rest perfusion and ${ }^{18} \mathrm{~F}$-FDG PET data; correction of the septum and the lateral wall was noted (Figs. 1E and 1F). The reconstructed images were displayed in short-axis, horizontal long-axis, and vertical long-axis views and studied for perfusion defects and ${ }^{18} \mathrm{~F}-\mathrm{FDG}$ uptake in corresponding areas.

The reconstructed slices from the first processing revealed an interchanged septum and lateral wall, and the interpretation was difficult. The second processing, with selection of prone positioning, allowed correct reorientation of the heart and correct positioning of the septum and the lateral wall. After comparing the matched rest perfusion and ${ }^{18} \mathrm{~F}-\mathrm{FDG}$ PET slices, we found perfusion to be absent from the mid- and basal anteroseptal and mid- and basal inferoseptal segments but ${ }^{18} \mathrm{~F}$-FDG uptake to be present in the same segments, suggesting viable myocardium (mismatched perfusion and metabolism) corresponding to the LAD territory. The patient underwent angioplasty with stenting of the LAD territory and is now symptom-free.

\section{Patient 2}

A 61-y-old woman with type II diabetes and hypertension complained of breathlessness on exertion over 6 months that worsened to the point that she had become breathless even at rest (New York Heart Association grade IV) for the past $15 \mathrm{~d}$. She did not complain of chest pain and had no history of myocardial infarction. The level of troponin $\mathrm{T}$ was 
normal. Two-dimensional echocardiography revealed dextrocardia with situs inversus, a reduced left ventricular ejection fraction of $30 \%$, and hypokinesia in the anterior wall segments, apex, anteroseptal segments, and anterolateral segments. Coronary artery angiography showed a type III LAD vessel with minor plaque followed by a $90 \%$ occlusive lesion; the left circumflex coronary artery was patent, and the right coronary artery was found to be the dominant artery, with minor plaque proximally and distally. The patient was referred for assessment of myocardial viability in the LAD territory.

The acquisition was done with parameters similar to those used in patient 1 . The reconstruction was also similarly done twice, to understand the differences in the reconstructed slices and the positioning of the myocardial walls (Fig. 2).

The second processing, done by choosing the prone position during data selection, allowed correct positioning

of the myocardial septum and lateral wall. The scan results showed absence of perfusion in the apex, apical septum, midand basal anterior walls, and mid- and basal anteroseptal segments. All these segments showed ${ }^{18}$ F-FDG uptake, suggesting viable myocardium (mismatched perfusion and metabolism) corresponding to the LAD territory. The patient underwent coronary bypass grafting of the LAD and right coronary arteries.

\section{Patient 3}

A 42-y-old man with hyperlipidemia and type II diabetes mellites presented with dyspnea on exertion (New York Heart Association grade II). He was on medical management for ischemic heart disease and later developed sudden-onset chest pain and worsening of breathlessness. Electrocardiography showed Q waves with right-bundle-branch block. Two-dimensional echocardiography showed mesocardia with a dilated left atrium, a left ventricular ejection fraction of $25 \%$, and

A
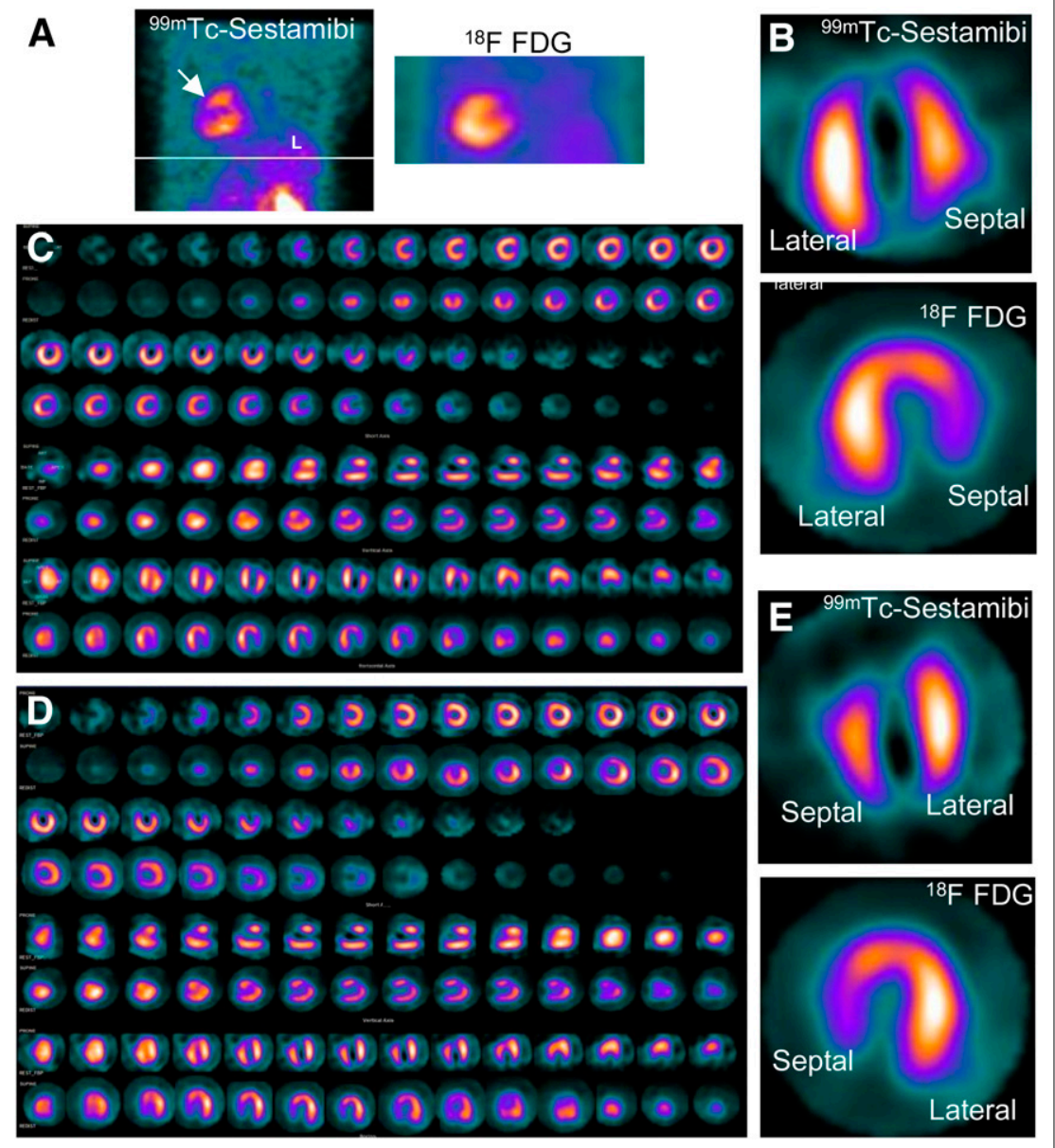

FIGURE 2. A 61-y-old woman had dextrocardia with situs inversus. (A) On maximumintensity projection, heart was seen in right chest and liver on left side (arrow). (B and $C)$ Processing of raw data in feet-first supine position showed interchanged lateral wall and septum in reconstructed 3 -axes display image (C) and horizontal long-axis image (B). (D and E) Orientation of septum and lateral wall was corrected in repeat processing (D), which was done after entering dataset for feet-first prone position instead of feet-first supine to match conventional nomenclature of display image (E). Perfusion was absent from apex, apical septum, mid- and basal anterior walls, and mid- and basal anteroseptal segments whereas ${ }^{18} \mathrm{~F}-\mathrm{FDG}$ uptake was present in same segments, suggesting viable myocardium (matched perfusion and metabolism). hypokinesia in the apex, septum, and anterior wall segments. A viability study was advised to assess myocardial involvement.

Resting perfusion images were also obtained in the feet-first supine position in a $180^{\circ}$ anticlockwise right-lateral-toleft-lateral arc, and ${ }^{18}$ F-FDG PET images were acquired using the same standard parameters as for patients 1 and 2 .

The raw data were reconstructed without changing the position of the patient in the dataset entered. The septum and the lateral wall were positioned normally (Fig. 3).

As orientation of cardiac chambers was not altered in this patient (mesocardia) as compared to the earlier 2 patients with dextrocardia, the modified processing using changed positioning of the patient was not required. The septum and lateral wall positioning matched the conventional nomenclature using regular positioning of the patient during processing of the raw dataset (Figs. 3C-3E).

The rest perfusion slices showed no perfusion in the apex or in the apical anterior, apical septal, apical inferior, apical lateral, basal anteroseptal, or midinferior segments and reduced perfusion in the entire anterior wall and septum. The ${ }^{18}$ F-FDG PET images showed no uptake in the myocardial segments in which perfusion was reduced or absent. Thus, the myocardium was not viable in the infarcted segments (matched perfusion and metabolism). 


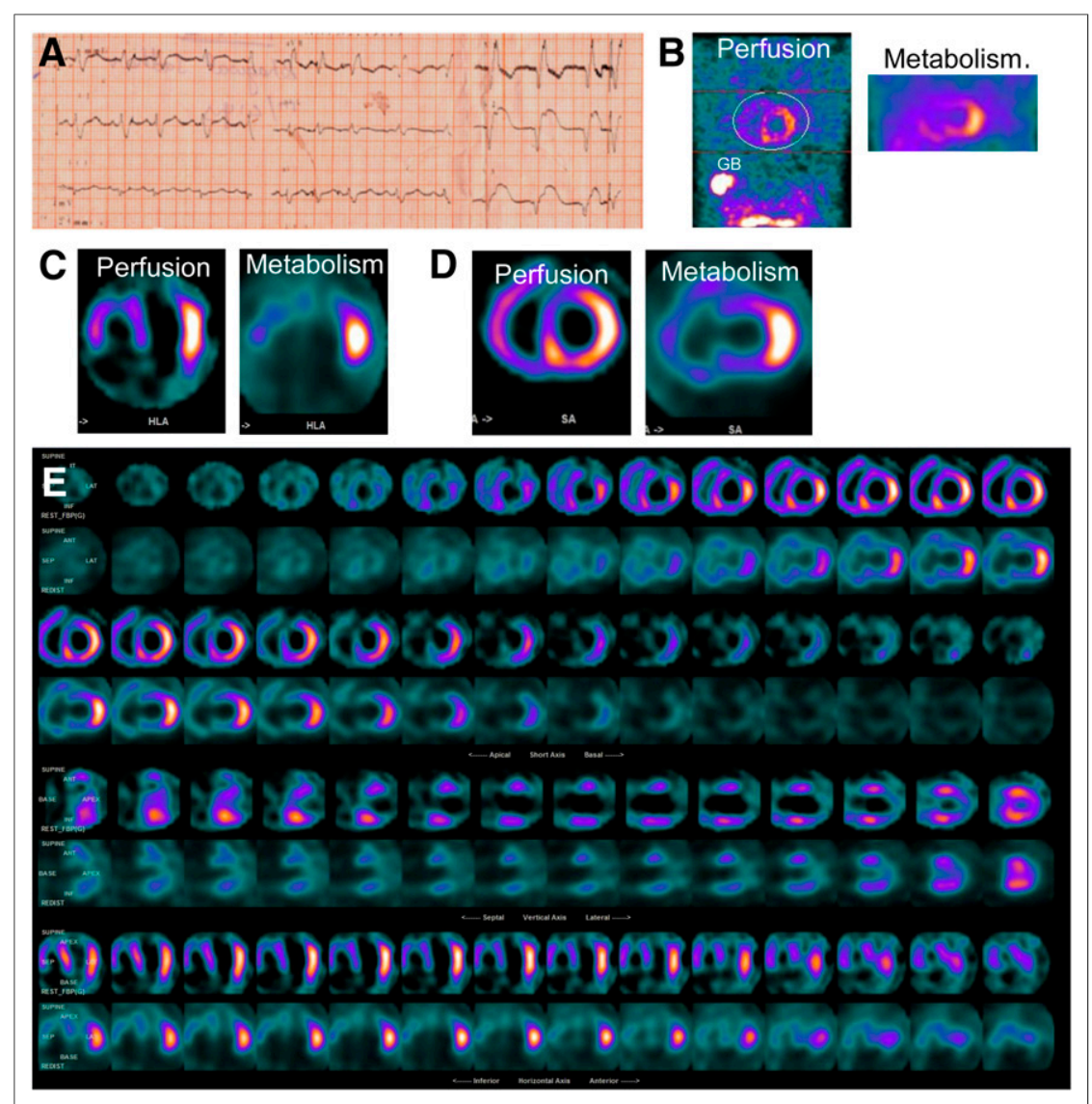

FIGURE 3. A 42-y-old man with mesocardia presented with breathlessness. Electrocardiography (A) showed ST-segment elevation in V4 and V5, Q waves in V1 and V4-V6, and right bundle branch block. (B) Maximum-intensity projections showed heart in midline of chest and gallbladder and liver on right side. (C-E) Processing of raw data in feet-first supine position showed lateral wall and septum positioned normally on reconstructed horizontal long-axis image (C) and short-axis image (D) and on display images (E). Rest perfusion slices showed absence of perfusion in apex, apical anterior, apical septal, apical inferior, apical lateral, basal anteroseptal, and mid-inferior segments. Perfusion was reduced in entire anterior wall and septum. ${ }^{18}$ F-FDG PET images did not show uptake in corresponding myocardial segments, suggesting absence of viable myocardium in infarcted segments (matched perfusion and metabolism).

\section{DISCUSSION}

According to the classification provided by Arcilla and Gasul (2), the first 2 cases of dextrocardia in our series were type I (dextrocardia with situs inversus) and the third was type IV (mesocardia).

Many approaches to the acquisition and processing of MPS images have appeared in the published literature. Complete dextroversion of the heart shows the anterior and inferior walls in the normal orientation but the septum and lateral wall interchanged and the right ventricle positioned to the left of the left ventricle. Thus, after processing and reconstruction of the raw data, the septum and lateral wall are seen to be interchanged.

Many case reports and imaging atlases mention use of an LAO-to-RPO arc for MPS acquisitions in dextrocardia and report an interchanged positioning of the walls. However, these reports have used the conventional nomenclature, keeping the interchanged walls in mind $(7,8,12,13)$. Slart et al. added CT-based attenuation correction, which helped in 2 ways: by correcting perfusion defects due to diaphragmatic attenuation and by allowing an understanding of the orientation of the heart chambers and walls (9). Özdemir et al. described 2 acquisition methods. The first was according to clinical routine protocols. SPECT was performed in a $180^{\circ}$ counterclockwise circular orbit beginning at $45^{\circ}$ RAO and ending at $45^{\circ} \mathrm{LPO}$ in a feet-first supine position. The first processing used normal parameters and the second used feet-first prone positioning so that the heart with dextrocardia was positioned as a normal heart would be. The second acquisition method applied by Özdemir et al. was according to dextrocardia protocols. SPECT was performed in a $180^{\circ}$ counterclockwise circular orbit beginning at $45^{\circ} \mathrm{LAO}$ and ending at $45^{\circ} \mathrm{RPO}$ in a head-first supine position, with the second processing using the dextrocardia parameters of the camera software (4). Ayeni et al. acquired MPS images of situs inversus totalis using feet-in supine positioning and 2 processing methods; the second method applied feet-first prone positioning, which was entered in the processing software and allowed the lateral wall and septum to be reconstructed in the normal axis (3). Qutbi et al. suggested a change from feet-in during acquisition to head-in or prone during processing to correct the orientation of the lateral wall and septum $(5,6)$.

Multiple methods can be used to acquire the studyplacing the patient either supine or prone with either the head first or the feet first and either selecting the appropriate protocol or choosing not to (3-9). However, use of multiple methods complicates the acquisition, processing, and interpretation of images and has been found to lead to artifacts $(12,13)$.

In view of the American Society of Nuclear Cardiology guidelines mentioning that the heart should be kept in the center of the arc of rotation (10), a clockwise acquisition from LAO to RPO with feet-first supine positioning appears to be the simplest method of acquiring MPS images in a patient who has dextrocardia with situs inversus. However, a right-lateral-to-left-lateral arc was used in our patient 3, who had mesocardia with the heart in a retrosternal position. 
Thus, it will be easy to adapt a practice of informing the technical staff about the altered arc of imaging, without changing the usual positioning of the patient (commonly, feet-first supine for a dual-head $\gamma$ camera). Because the protocol for cardiac ${ }^{18} \mathrm{~F}$-FDG PET acquires counts in a $360^{\circ}$ arc, no additional modifications are required.

We find that changing the position of the patient from supine during acquisition to prone during processing is the best way of reorienting the heart walls to match the conventional display nomenclature. Such a change corrects the orientation of the walls in both the SPECT data (acquired in a $180^{\circ}$ arc) and the PET data (acquired in a $360^{\circ}$ arc), making processing and interpretation easy. However, in dextrocardia cases for which the entire heart is in the middle of the chest (mesocardia) but the orientation of the chambers is unchanged (the right ventricle is to the right of the left ventricle), the images are to be processed as usual.

Whatever the patient positioning and acquisition method used, it is essential to know the exact details of the orientation of the heart and its chambers so that correct interpretation of the images is possible.

\section{CONCLUSION}

For imaging of myocardial viability in the rare cardiac malposition of dextrocardia, the simpler approach is to acquire images using the same position as in patients with a normally oriented heart (commonly, feet-first supine for a dual-head $\gamma$-camera), changing just the direction of the arc during acquisition. However, if prone positioning is selected during processing of rest perfusion and ${ }^{18} \mathrm{~F}$-FDG PET images, the conventional nomenclature for the walls can be met, allowing easy interpretation of perfusion defects and myocardial viability.

\section{DISCLOSURE}

No potential conflict of interest relevant to this article was reported.

\section{ACKNOWLEDGMENT}

Dr. Pamela Moyade, senior resident, Seth G.S. Medical College and King Edward Memorial Hospital, helped obtain and compile the information from the 2 patients but did not contribute to the writing, data analysis, or review of this article.

\section{REFERENCES}

1. Maldjian PD, Saric M. Approach to dextrocardia in adults. AJR. 2007; 188(suppl);S39S49.

2. Arcilla RA, Gasul BM. Congenital dextrocardia: clinical, angiocardiographic, and autopsy studies on 50 patients. J Pediatr. 1961;58:251-262.

3. Ayeni OA, Malan N, Hammond EN, Vangu MD. Myocardial perfusion SPECT imaging in dextrocardia with situs inversus: a case report. Asia Ocean J Nucl Med Biol. 2016;4:109-112.

4. Özdemir S, Gazi E. Myocardial perfusion SPECT imaging in dextrocardia: a case report. Mol Imaging Radionucl Ther. 2013;22:70-72.

5. Qutbi M. SPECT myocardial perfusion imaging in patients with dextrocardia. J Nucl Cardiol. 2019;26:1197-1204.

6. Qutbi M, Soltanshahi M, Ansari M, Hashemi H, Neshandar Asli I, Shafiei B. Quantitation in dextrocardia on myocardial perfusion imaging: how to perform quantitative analysis using Cedars-Sinai software. Nucl Med Rev Cent East Eur. 2018;21:50-52.

7. Kashyap R, Abrar ML, Bhattacharya A, Rohit MK, Mittal BR. Myocardial perfusion scintigraphy in a case of dextrocardia: doing it "right." Indian J Nucl Med. 2012;27:252-253.

8. Turgut B, Kitapci MT, Temiz NH, Unlü M, Erselcan T. Thallium-201 myocardial SPECT in a patient with mirror-image dextrocardia and left bundle branch block. Ann Nucl Med. 2003;17:503-506.

9. Slart RH, de Boer J, Jager PL, Piers DA. Added value of attenuation-corrected myocardial perfusion scintigraphy in a patient with dextrocardia. Clin Nucl Med. 2002;27:901-902.

10. Dorbala S, Ananthasubramaniam K, Armstrong IS, et al. Single photon emission computed tomography (SPECT) myocardial perfusion imaging guidelines: instrumentation, acquisition, processing, and interpretation. J Nucl Cardiol. 2018;25: 1784-1846.

11. Dilsizian V, Bacharach SL, Beanlands RS, et al. ASNC imaging guidelines/ SNMMI procedure standard for positron emission tomography (PET) nuclear cardiology procedures. J Nucl Cardiol. 2016;23:1187-1226.

12. Fathala A. Myocardial perfusion single photon computed tomography: an atlas. J Saudi Heart Assoc. 2011;23:107-122.

13. Muzaffar R, Raslan O, Ahmed F, Goldfarb L, Sterkel B, Osman MM. Incidental findings on myocardial perfusion SPECT images. J Nucl Med Technol. 2017;45:175180. 